\title{
Sintomatología obstructiva en mujer joven por hidrosalpinx izquierdo
}

\author{
Luján Marco S, Bango García V, Gimeno Argente V, Bosquet Sanz M, Arlandis Guzman S, \\ Jiménez Cruz JF.
}

Servicio de Urología. Hospital Universitario La Fe. Valencia.

Actas Urol Esp. 2008;32(2):246-248

\section{RESUMEN}

SINTOMATOLOGÍA OBSTRUCTIVA EN MUJER JOVEN POR HIDROSALPINX IZQUIERDO

Se describe el caso de una mujer de 43 años con sintomatología de llenado y vaciado tras realización de histerectomía con estudio urodinámico compatible con obstrucción infravesical. Se diagnosticó un hidrosalpinx izquierdo que fue tratado quirúrgicamente tras el cual la paciente quedo asintomática.

Palabras clave: Síntomas obstructivos en mujer. Hidrosalpinx.

\section{ABSTRACT \\ OBSTRUCTIVE SYMPTOMS IN YOUNG WOMAN BY LEFT HIDROSALPINX}

We describe the case of a 43 years old woman with obstructive and irritative symptoms after having an hysterectomy. Bladder outlet obstruction was observed in an urodinamyc study. She was later diagnosed with left hidrosalpinx. Salpingectomy was performed, as a result all symptoms have disappear.

Keywords: Obstructive symptoms in woman. Hidrosalpinx.

$\mathrm{L}$ a obstrucción urinaria en la mujer está probablemente infradiagnosticada por diversas causas $^{4}$. El objetivo de esta nota clínica es aportar un nuevo caso a la literatura, ya que el origen de la obstrucción en esta paciente es inusual.

\section{CASO CLÍNICO}

Mujer de 43 años remitida a la consulta de Neurourología-Urodinámica desde Ginecología para valoración y realización de estudio urodinámico por dificultad a la micción, junto con sindrome de frecuencia/urgencia poshisterectomía de útero miomatoso hace 2 años. La paciente no presenta antecedentes médicos de interés ni toma medicación actualmente. Como antecedente obstétrico destaca una gestación con cesárea. Clinicamente refiere sintomas de llenado con una frecuencia miccional diurna aumentada (8-10) y nocturia (3), con urgencia miccional sin escape. Como síntomas de vaciado destaca disuria con chorro entrecortado junto con sensación de resi- duo. No refiere infecciones del tracto urinario, hematuria, ni incontinencia de esfuerzo.

A la exploración física presenta un buen estado general con abdomen blando y depresible sin evidenciar masas ni visceromegalias. Exploración neuro-urológica dentro de la normalidad. A nivel vaginal no se visualizan celes ni escapes con esfuerzo, apreciándose al tacto aumento de consistencia sobre cara lateral izquierda

Se realiza estudio urodinámico (Cistomanometría más EMG) (Fig. 1) presentando en fase de llenado, un primer deseo a los $100 \mathrm{~mL}$ con deseo miccional precoz (120 mL), siendo la capacidad cistomanométrica máxima de $400 \mathrm{~mL}$, produciéndole dolor. Durante la fase de vaciado el flujo máximo es bajo (6 mL/seg) con presión del detrusor elevadas (Pdet-Qmax $52 \mathrm{~cm}$ de $\mathrm{H} 2 \mathrm{O}$ y Pdet $\max 72 \mathrm{~cm}$ de $\mathrm{H} 2 \mathrm{O}$ ), buena relajación del esfinter externo y ausencia de residuo posmiccional. Todos estos parámetros serian compatibles con obstrucción infravesical al flujo urinario. 


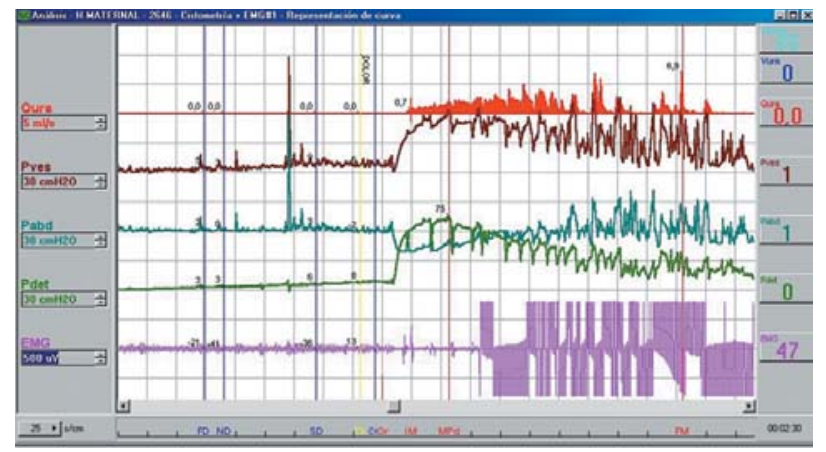

FIGURA 1. Estudio urodinámico donde destaca una fase de vaciado con Pdet (segunda linea por abajo) elevadas al inicio de la micción, con flujo disminuido (primera línea por arriba) y silencio electromiográfico (línea de la parte inferior). Se produce artefacto en EMG al final de la micción por caida de electrodo.

Se prescribe tratamiento empírico con alfa-bloqueantes (tamsulosina 0,4 mg/24 hora). Se solicita urinocultivo con resultado negativo; análisis con hematología y bioquímica dentro de la normalidad. La ecografia renal bilateral y vesical muestra Riñones de ecoestructura y morfología dentro de la normalidad. Vejiga con repleción satisfactoria, de contornos lisos existiendo un tabique que aparentemente la divide en dos porciones, sin evidenciar punto claro de conexión (Fig. 2). Tras la micción persisten los dos componentes. En nuestra consulta realizamos ecografia transvaginal cateterizando vejiga con sondaje uretral, comprobando la localización de la misma en vejiga con una imagen anecoica adyacente sin aparente comunicación vesical.

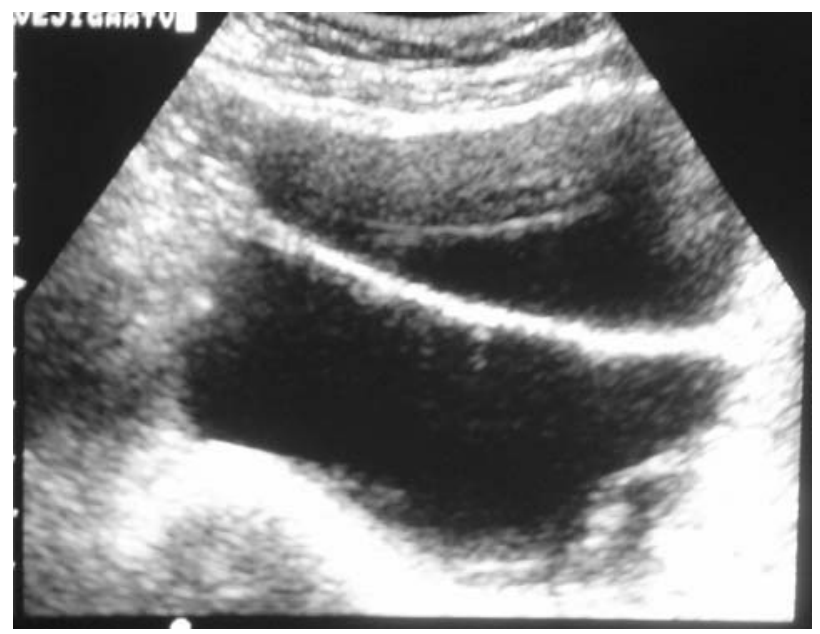

FIGURA 2. Ecografía transabdominal sobre región hipogástrica donde se observan dos estructuras anecoicas en teórica localización de vejiga urinaria.
Ante el hallazgo de estructura yuxtavesical de características quísticas solicitamos RMN Pélvica (Fig. 3) apreciándose en íntima relación con anexo izquierdo masa quística de aproximadamente $10 \times 8 \mathrm{~cm}$ de diámetros máximos sugestiva de hidrosalpinx marcadamente distendido que condiciona compresión de la vejiga.

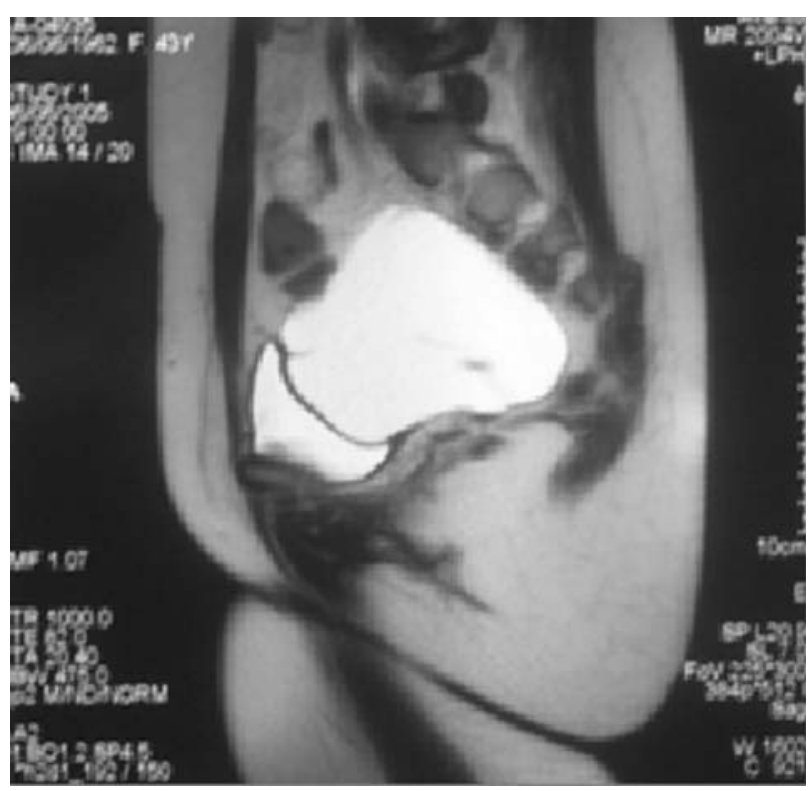

FIGURA 3. Secuenciada en T1. Sección sagital donde se observa compresión vesical por masa quistica sobre anexo izquierdo.

La paciente es remitida a ginecología para su valoración, persistiendo la clínica a pesar del tratamiento con alfa-bloquentes. Se realiza salpinguectomía izquierda vía laparoscópica por hidrosalpinx.

Es valorada a los 3 meses de la cirugía. La paciente refiere desaparición del síndrome frecuencia/urgencia con flujo continuo, sin disuria ni sensación de residuo vesical. Se realiza una flujometria libre (Fig. 4) con un volumen de vaciado de $573 \mathrm{~mL}$, flujo máximo 29,6 mL/seg., tiempo de vaciado de 68 segundos y residuo posmiccional de $30 \mathrm{~mL}$.

\section{DISCUSIÓN}

La primera descripción de obstrucción orgánica del tracto urinario inferior en la mujer fue dada por Ambrosio Paré en $1575^{1}$. La obstrucción del tracto urinario inferior se diagnostican con mucha menos frecuencia en la mujer que en 


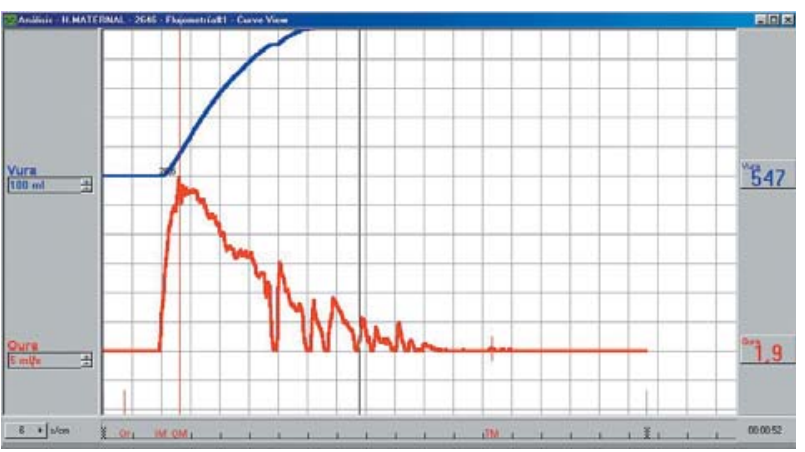

FIGURA 4. Flujometria libre postratamiento donde se evidencia mejoria del flujo urnario.

el hombre, con una incidencia estimada de alrededor del 2,7-8\% ${ }^{5}$. No existe una definición estándar, ni consenso para el diagnóstico de obstrucción urinaria infravesical y, los nomogramas aplicados en los hombres no son válidos en mujeres porque presentan presiones del detrusor más bajas durante el vaciado. Diversos grupos consideran unos márgenes de Q max por debajo de 10$15 \mathrm{~mL} / \mathrm{seg}$ y una Presión del detrusor al flujo máximo por encima de $20-35 \mathrm{~cm}$ de agua ${ }^{3,7}$ siendo los de nuestra paciente compatibles con estos. Para Chassagne et al. ${ }^{8}$ la Qmax debe ser menor o igual a $15 \mathrm{ml} / \mathrm{seg}$ y PdetQmax mayor o igual de $21 \mathrm{~cm}$ de agua con una sensibilidad del 74,3\% y una especificidad del 91,1\%, mientras que para Lemack et al. ${ }^{9}$ la Qmax debe ser igual o menor a $11 \mathrm{ml} / \mathrm{seg}$, PdetQmax mayor o igual a $21 \mathrm{~cm}$ de agua con una sensibilidad del 91,5\% y especificidad del 73,6\%. Se suelen asociar estudios videourodinámicos para observar alteraciones de cuello-uretra durante el vaciado. Para Axelrod et al. ${ }^{10}$ se definiría la obstrucción con una Qmax por debajo de $12 \mathrm{ml} / \mathrm{seg}$, PdetQmax superior a 20 $\mathrm{cm}$ de agua junto con estudio videourodinámico que evidencie la obstrucción del cuello vesical.

Las causas de la obstrucción urinaria infravesical en la mujer son más numerosas y variadas que en el hombre, dividiéndose básicamente en orgánicas y funcionales ${ }^{5}$. En la paciente descartamos la disinergia detrusor-esfinter externo al producirse un silencio electromiográfico durante el estudio de presión/flujo. Las causas anatómicas más frecuentes son el prolapso de órganos pélvicos (rectocele, cistocele) y la obstrucción tras cirugía de la incontinencia ${ }^{6}$, dos circunstancias que no presentaba la paciente. Los procesos ginecológicos producen obstrucción urinaria por mecanismo compresivo extrínseco, principalmente el carcinoma de cuello uterino y vaginal y las masas ováricas ${ }^{2}$. El hidrosalpinx es una dilatación de la trompa de Falopio por material líquido aséptico, cuyo crecimiento provocó, en esta paciene de manera inusual, la sintomatología del tracto urinario inferior.

El tratamiento empírico con alfa-boqueantes no resolvió la clínica, mientras que sí lo hizo la salpinguectomía por vía laparoscópica, objetivándose normalización de la flujometría.

\section{REFERENCIAS}

1. Ramos C, Leiva O. "Retención urinaria en la mujer. Urodinámica clínica”. Vector ediciones. 1989;143.

2. Salinas J, Romero J. "Urodinámica clínica: Disfunciones vesicouretrales". Luzan ediciones .2002; 188.

3. Kuo HC. Urodynamic parameters for the diagnosis of bladder outlet obstruction in women. Urol Int. 2004;72(1):4651.

4. Groutz A, Blaivas JG, Chaikin DC. Bladder outlet obstruction in women: definition and characteristics. Neurourol Urodyn. 2000;19(3):213-220.

5. Mc Crery R, Apell R. Bladder Outlet Obstruction in Women: Iatrogenic, Anatomic, and Neurogenic. Curr Urol Rep.2006;7(5):363-369.

6. Nitti VW, Tu LM, Gitlin J. Diagnosing bladder outlet obstruction in women. J Urol. 1999;161(5):1535-1540.

7. Defreitas GA, Zimmern PE, Lemack GE, Shariat SF. Refining diagnosis of anatomic female bladder outlet obstruction: comparison of pressure-flow study parameters in clinically obstructed women with those of normal controls. Urology. 2004;64;(4):675-679; discussion 679-681.

8. Chassagne S, Bernier PA, Haab F, Roehrborn CG, Reisch JS, Zimmern PE. Proposed cutoff values to define bladder outlet obstruction in women. Urology. 1998;51(3):408411.

9. Lemack GE, Zimmern PE. Pressure flow analysis may aid in identifying women with outflow obstruction. J Urol. 2000;163(6): 1823-1828.

10. Axelrod SL, Blaivas JG. Bladder neck obstruction in women. J Urol. 1987;137(3):497-499.

Correspondencia autor: Dr. S. Luján Marco

Servicio de Urologia. Hospital Universitario La Fe

Avda. Campanar, 21 - 46009 Valencia

Tel.: 963862700

E-mail: slujanmarco@comv.es

Información artículo: Nota Clínica

Trabajo recibido: junio 2007

Trabajo aceptado: noviembre 2007 